\title{
Efectos de la exposición al humo de tabaco ambiental en no fumadores
}

\author{
SERGIO BELLO S.*, SUSANA MICHALLAND H.*, MARINA SOTO I.**, \\ CARLA CONTRERAS N.*** y JUDITH SALINAS C.****
}

\section{EFFECTS IN PASSIVE SMOKERS OF ENVIRONMENTAL TOBACCO SMOKE EXPOSURE}

\begin{abstract}
National and international environmental tobacco smoke studies were reviewed regarding its effects on passive smokers' health. Environmental tobacco smoke is composed by more than 4,000 known substances, which are different if they are in the mainstream or sidestream. Studies with biomarkers like cotinine have concluded that exposed nonsmokers have an increased cardiovascular morbi-mortality. Passive smokers also have an increased risk of lung, nasal sinus and breast cancer. Children have more acute and chronic respiratory symptoms, low respiratory infections, otitis and sudden infant death syndrome. Environmental tobacco smoke induces asthma and causes exacerbations in both children and adults. In reproductive health, it decreases the age of menopause and produces menstrual disorders. Newborn infants of passive smoking mothers have a higher probability of low birth weight and to be small for gestational age. This international scientific evidence should lead countries to take measures in order to protect nonsmokers' health.
\end{abstract}

Key words: Environmental tobacco smoke, passive smoking, nonsmokers', heath.

\section{RESUMEN}

Se realizó una revisión de los estudios nacionales e internacionales sobre los efectos del humo de tabaco ambiental en la salud de los fumadores pasivos. El humo de tabaco ambiental está conformado por más de 4.000 sustancias químicas, las que difieren cuali y cuantitativamente al estar presentes en la corriente principal $o$ en la lateral. Los no fumadores expuestos presentan un aumento de morbimortalidad por enfermedad cardiovascular, incluso con estudios con biomarcadores como cotinina. Además los fumadores pasivos tienen un riesgo aumentado de presentar cáncer de pulmón, de senos paranasales y mama. En niños existe un mayor número de síntomas respiratorios agudos y crónicos, infecciones respiratorias bajas, otitis media y síndrome de muerte súbita infantil. El humo de tabaco ambiental favorece la inducción y exacerbación de asma bronquial en niños y adultos. En la salud reproductiva, disminuye la edad de aparición de la menopausia y produce desórdenes menstruales. Los recién nacidos hijos de madres fumadoras pasivas tienen mayor probabilidad de nacer con bajo peso o de ser pequeños para la edad gestacional. La evidencia científica internacional debe contribuir a que los países tomen medidas para proteger la salud de los no fumadores. salud.

Palabras clave: Humo de tabaco ambiental; fumadores pasivos; daños a la

\footnotetext{
Instituto Nacional del Tórax. Departamento Políticas Públicas Saludables. Ministerio de Salud:

* Médico neumólogo.

** Enfermera.

*** Estudiante $5^{\circ}$ año, Escuela de Medicina, Universidad de Chile.

$* * * *$ Asistente social.
} 


\section{INTRODUCCIÓN}

Una gran cantidad de datos toxicológicos, experimentales y epidemiológicos, recolectados a partir de la década de 1950, han establecido que el consumo de tabaco es la mayor causa prevenible de morbilidad y mortalidad en el mun$\mathrm{do}^{1}$, produciendo más de 5 millones de muertos cada año. En Chile son 14.000 los fallecidos anualmente ${ }^{2}$.

Desde la década de 1970, la exposición al humo de tabaco ambiental (EHTA) ha sido estudiada como factor de riesgo para diversas enfermedades y se ha identificado como una causa prevenible de morbilidad y mortalidad entre los no fumadores. Durante los ' 80 se publicaron numerosas revisiones sobre los efectos de fumar pasivamente. Esto incluye el informe del Cirujano General de EE.UU (1986) ${ }^{3}$, del Consejo Nacional de Investigación de los Estados Unidos del mismo año ${ }^{4}$ y del Consejo Nacional de Salud e Investigación Médica de $\operatorname{Australia}^{5}$ y culminó en una importante revisión realizada por la Agencia de Protección del Medio Ambiente (EPA) de EE.UU en $1992^{6}$.

Más recientemente se han continuado publicando revisiones muy importantes. Dentro de ellas destacan los informes del Comité Científico en Tabaco y Salud del Reino Unido ${ }^{7}$, de la Agencia Internacional de Investigación en Cáncer (IARC) ${ }^{8}$ y de la Agencia de Protección del Medio Ambiente de California (EPA, California) en 1997 y $2005^{9,10}$.

La toma de conciencia sobre los numerosos y graves daños para la salud producidos por la EHTA ha hecho plantear a las autoridades de salud y los gobiernos, políticas públicas para proteger la salud de la población del fumar involuntario. El Convenio Marco para el Control del Tabaco, tratado internacional auspiciado por la
$\mathrm{OMS}^{11}$, contempla, entre otras medidas, la protección del fumador pasivo. Este convenio fue ratificado por Chile en Marzo de 2005.

Se ha estimado conveniente realizar una revisión de los efectos de la EHTA en la salud, considerando la literatura de los últimos 15 años a nivel nacional e internacional, con el propósito de contribuir a su divulgación médica y pública. Con ello se espera aportar al cambio en la percepción social del tabaco, para que su consumo público sea rechazado y favorecer las políticas de protección de los no fumadores.

\section{CONSTITUYENTES DEL HUMO DE TABACO Y EVALUACIÓN DE LA EXPOSICIÓN}

El humo del tabaco es una mezcla compleja de gases y partículas que contienen más de 4.000 sustancias químicas. Hay dos clases de humo de tabaco: la corriente principal, que es la que aspira el fumador hacia sus pulmones al inhalar el cigarrillo, y la corriente secundaria o lateral, que es el humo que se desprende hacia el ambiente desde la punta encendida, entre bocanada y bocanada (Figura 1).

El humo de tabaco ambiental (HTA) es el humo que queda en suspensión en un ambiente donde hay personas fumando. El $85 \%$ proviene de la corriente lateral del humo de cigarrillo en combustión y el otro $15 \%$ corresponde al humo de la corriente principal espirado por los fumadores activos.

La exposición al humo de tabaco en los fumadores pasivos y en los activos es diferente en términos cuantitativos y cualitativos ${ }^{12}$. Debido a que las temperaturas son más bajas en el cono ardiente del cigarrillo entre aspiraciones, la mayoría de los productos de pirólisis parcial son más altos en el humo lateral en comparación

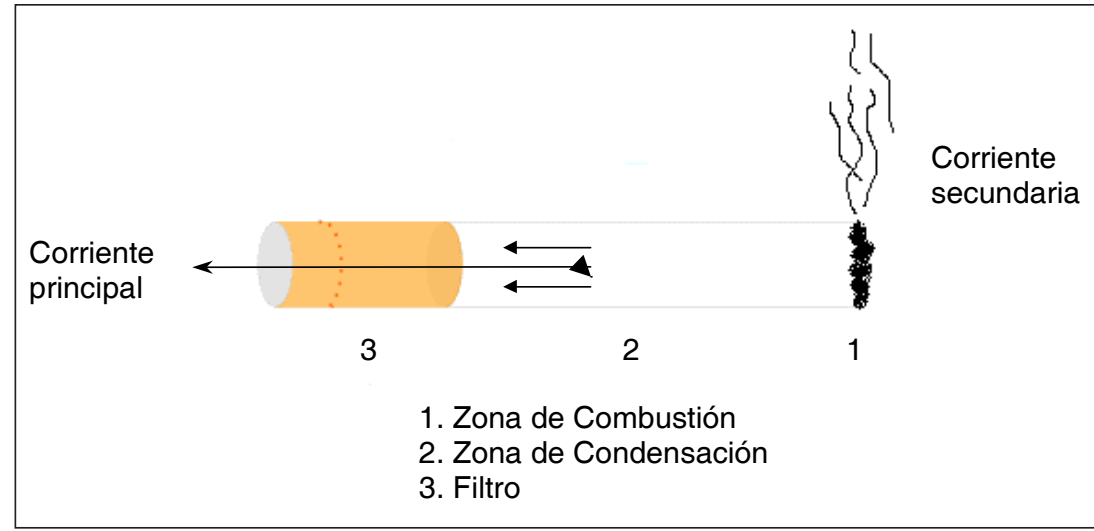

Figura 1. Tipos de humo de tabaco. 
con el humo principal. En consecuencia, el humo de la corriente lateral tiene mayores concentraciones de algunas sustancias tóxicas y carcinogénicas aunque la dilución provocada por el aire ambiental reduce marcadamente las concentraciones inhaladas por el fumador pasivo en comparación con las inhaladas por el fumador acti$\mathrm{vo}^{4,6,9}$.

Por lo tanto el consumo de tabaco en ambientes cerrados aumenta en éstos los niveles de partículas respirables, nicotina, monóxido de carbono (CO), acroleína, formaldehído, y muchas otras (Tabla 1). Se han encontrado además más de 40 sustancias químicas que producen cáncer en animales y en el ser humano, siendo las principales los hidrocarburos aromáticos policíclicos, aminas aromáticas, benceno, arsénico, etc.

La detección de componentes del humo de tabaco o sus metabolitos en los líquidos corpo- rales o en el aire alveolar proporciona evidencia de exposición al humo del tabaco, tanto en fumadores activos como en los pasivos.

En la actualidad, los marcadores más sensibles y específicos para la exposición al humo del tabaco son la nicotina y su metabolito, cotinina $^{4}$. Ésta se forma por oxidación de la nicotina por el citocromo P-450, metabolizándose extensamente, y sólo el $17 \%$ de la misma se elimina inalterada en la orina.

Debido a que la vida media de la nicotina circulante es generalmente menor a 2 horas $^{13}$, las concentraciones de nicotina en los líquidos corporales reflejan exposiciones recientes. En contraste, la cotinina tiene una vida media de alrededor de 10 horas en la sangre de los fumadores $\operatorname{activos}^{14}$, por lo que proporciona información acerca de una exposición más prolongada al humo de tabaco, siendo el biomarcador

Tabla 1. Principales componentes del humo de tabaco ambiental y sus efectos sobre la salud

\begin{tabular}{|c|c|c|}
\hline Principales componentes & $\begin{array}{l}\text { Efecto carcinogénico } \\
\text { (IARC)* }\end{array}$ & Efectos en la salud (no cáncer) \\
\hline \multicolumn{3}{|l|}{ Fase gaseosa } \\
\hline Acetaldehído & $2 \mathrm{~B}$ & Irritante, dermatitis \\
\hline Acetona & & Irritante, mareos \\
\hline Acroleína & 3 & Irritante, edema pulmonar \\
\hline Benceno & 1 & Depresor SNC, náuseas \\
\hline Monóxido de carbono & & Cefalea, mareos \\
\hline Formaldehído & $2 \mathrm{~A}$ & Irritante, inductor de asma \\
\hline Metanol & & Neurotóxico, irritante \\
\hline N-Nitrosodietilamina & $2 \mathrm{~A}$ & \\
\hline N-Nitrosodimetilamina & $2 \mathrm{~A}$ & Daño hepático \\
\hline Estireno & $2 \mathrm{~B}$ & Depresor SNC, irritante \\
\hline \multicolumn{3}{|l|}{ Fase particulada } \\
\hline 2-Naftilamina & 1 & Irritante, mareos \\
\hline 4-Aminobifenil & 1 & Hematuria \\
\hline Arsénico & 1 & Hemólisis, neuropatía \\
\hline Benzo(a)antraceno & $2 \mathrm{~A}$ & \\
\hline Benzo(a)pireno & $2 \mathrm{~A}$ & Dermatitis, irritante \\
\hline Cadmio & $2 \mathrm{~A}$ & Bronquitis, irritante \\
\hline Cromo & 1 & Toxicidad renal, hemólisis \\
\hline Plomo & $2 \mathrm{~B}$ & Alteraciones SNC, depresión \\
\hline Níquel & 1 & Alteraciones inmunológicas \\
\hline Nicotina & & Adicción \\
\hline
\end{tabular}

* Clasificación de la IARC: Internacional Agency for Research on Cancer.

1. Carcinógeno para seres humanos.

2A. Carcinógeno probable para seres humanos, con evidencias suficientes en animales pero no en seres humanos.

2B. Carcinógeno posible para seres humanos, con evidencias limitadas en animales y no en seres humanos.

3. No clasificable como carcinógeno para seres humanos. 
más importante para distinguir entre fumadores, no fumadores expuestos y no fumadores no expuestos al humo de tabaco ambiental, y su grado de exposición ${ }^{10}$. Sin embargo, como método indirecto de exposición, la medición de nicotina en el ambiente se ha convertido en el método más usado, por su sencillez y costo. También se puede medir la concentración de tiocianato en los líquidos corporales, el $\mathrm{CO}$ en el aire exhalado y el nivel de carboxihemoglobina, pero no son tan sensibles y específicos como la cotinina para evaluar la EHTA ${ }^{10,15}$.

\section{EFECTOS SOBRE LA SALUD \\ DE LA EXPOSICIÓN AL HUMO DE TABACO AMBIENTAL}

La mayoría de los no fumadores sienten molestias cuando están expuestos al humo de tabaco. Pueden experimentar irritación de ojos, dolor de cabeza, mareos, cansancio, dolor faríngeo, náuseas, tos o dificultad respiratoria. Mientras los fumadores están habituados a los efectos irritantes de algunas sustancias presentes en el HTA, los no fumadores son afectados notoriamente al estar expuestos al mismo.

En la Tabla 2 se presentan los daños de la EHTA más importantes sobre la salud de las personas que se desarrollarán con más detalles a continuación. En la Tabla 3 se observan datos cuantitativos de riesgos asociados a la EHTA en EEUU y en el Reino Unido ${ }^{10,16}$, que permiten dimensionar la gran magnitud de dichos riesgos.

\section{Efectos cardiovasculares}

Se han propuesto al menos 5 factores por los cuales la EHTA contribuye a las manifestaciones clínicas de las enfermedades cardiovas- culares: inducción de ateroesclerosis, trombosis, espasmos de las arterias coronarias, arritmias cardíacas y disminución de la capacidad de transporte de oxígeno en la sangre. El efecto agudo más importante es el afecto arritmogénico, además de favorecer los procesos trombóticos. Esto es facilitado por el aumento del fibrinógeno plasmático y cambios en el perfil lipídico: disminución del colesterol HDL, peroxidación del LDL y un aumento de la relación colesterol total/ colesterol HDL. Por otra parte se ha encontrado un aumento de la agregación plaquetaria y disfunción endotelial, que también favorece el proceso ateroesclerótico y trombogénico ${ }^{17,18}$. Según los mecanismos involucrados, estos efectos pueden manifestarse en forma aguda o requerir de tiempos más prolongados.

En los fumadores pasivos, la absorción de muy bajas concentraciones del humo de tabaco afecta el sistema circulatorio sin existir una relación lineal, por lo cual el riesgo cardiovascular es mayor de lo esperado, lo que podría explicarse por el aumento de la agregación plaquetaria. Se ha observado que tan sólo 20 minutos de exposición al HTA (en cantidades habituales a las encontradas en un bar o restaurant) aumentan la agregación plaquetaria a niveles similares a los de un fumador activo ${ }^{19}$. Se ha estimado que el riesgo cardiovascular asociado a la EHTA es de un tercio de los fumadores activos.

En los últimos años han sido publicados varios meta-análisis y revisiones (Tabla 4) sobre el riesgo de cardiopatía coronaria y EHTA $^{20-22}$. Wells ${ }^{20}$ analizó 8 estudios en el lugar de trabajo y encontró que el riesgo relativo de cardiopatía coronaria varió entre 0,66 y 1,85 y el resumen del estudio poblacional mostró un riesgo relativo de 1,18 (IC 95\% 1,04-1,34).

La EHTA en el hogar fue estudiada en un

\section{Tabla 2. Efectos nocivos de la Exposición a Humo de Tabaco Ambiental sobre la salud, con evidencias concluyentes}

\begin{tabular}{|c|c|}
\hline Cardiovasculares & $\begin{array}{l}\text { Aumento mortalidad por enfermedades cardíacas. Aumento de morbilidad } \\
\text { por cardiopatía coronaria aguda y crónica }\end{array}$ \\
\hline Respiratorios & $\begin{array}{l}\text { Síntomas respiratorios crónicos en niños. Infecciones respiratorias bajas en } \\
\text { niños. Asma bronquial: inducción y exacerbación en niños y adultos. Otitis } \\
\text { media en niños }\end{array}$ \\
\hline Cáncer & Pulmonar, senos nasales y mama \\
\hline Salud Reproductiva & $\begin{aligned} \text { Recién nacidos: - } & \text { Bajo peso de nacimiento } \\
\text { - } & \text { Aumento de recién nacidos pequeños para la edad } \\
& \text { gestacional }\end{aligned}$ \\
\hline Síndrome Muerte Súbita Infantil & Aumento de morbilidad y mortalidad \\
\hline
\end{tabular}


Tabla 3. Riesgos anuales por la Exposición a Humo de Tabaco Ambiental en EEUU y Gran Bretaña

\begin{tabular}{|c|c|c|}
\hline & Estados Unidos* & Gran Bretaña** \\
\hline Cardiovasculares: & 46.000 & \\
\hline Rango muertes cardiopatía coronaria & $22.700-69.600$ & $7.264-12.868$ \\
\hline \multicolumn{3}{|l|}{ Respiratorias: } \\
\hline Asma (niños): episodios & 202.300 & \\
\hline nuevos casos & $8.000-26.000$ & $1.660-5.396$ \\
\hline Otitis Media: visitas & 789.700 & 135.943 \\
\hline \multicolumn{3}{|l|}{ Cáncer Pulmonar } \\
\hline Muertes & 3.400 & 623 \\
\hline Partos de pretérmino & 71.900 & \\
\hline Niños con bajo peso nacimiento & 24.300 & 3.805 \\
\hline \multicolumn{3}{|l|}{ Síndrome muerte súbita infantil: } \\
\hline Muertes & 430 & 208 \\
\hline
\end{tabular}

*EPA California ${ }^{9,10}$

**Action on Smoking and Health-UK ${ }^{16}$

Tabla 4. Algunos estudios de la Exposición a Humo de Tabaco Ambiental y Cardiopatía Coronaria

\begin{tabular}{lll}
\hline Estudio (referencia) & Tipo de estudio & OR o RR (IC 95\%) \\
\hline Wells et al (20) & $\begin{array}{l}\text { Meta análisis } \\
\text { 8 estudios, exposición en lugar de trabajo }\end{array}$ & $\begin{array}{l}1,28(1,20-1,37) \\
\text { Morbilidad: } 1,49(1,29-1,78) \\
\text { Mortalidad: } 1,23(1,14-1,32)\end{array}$ \\
Law et al (21) & Meta análisis & $\begin{array}{l}1,30(1,22-1,38) \\
\text { Ajustado por dieta: 1,23 (1,14-1,33) }\end{array}$ \\
He et al (22) & Meta análisis 18 estudios & $1,26(1,16-1,38)$ \\
Thun et al (23) & Meta análisis 17 estudios, exposición & $1,25(1,17-1,33)$ \\
Ciruzzi et al (24) & Caso-control Infarto agudo miocardio & $1,68(1,20-2,37)$ \\
Enstrom y Kabat (25) & Cohorte & $0,94(0,85-1,05)$ \\
Whincup et al (26) & Cohorte & $1,57(1,08-2,28)$ \\
\hline
\end{tabular}

meta análisis por Thun et $\mathrm{al}^{23}$ que incluyó 17 estudios. El riesgo de cardiopatía coronaria para el cónyuge no fumador aumentó en $25 \%$ (IC $95 \%$ 1,17-1,33) si el otro cónyuge fuma en la casa. El riesgo relativo de eventos fatales y no fatales fue 1,22 (IC 95\% 1,14-1,30) y 1,32 (IC $95 \%$ 1,04-1,67). Los individuos con otros factores de riesgo cardiovascular (como diabetes, dislipidemia, antecedentes de infarto cardíaco familiar o personal) son más susceptibles a la EHTA según el estudio de Ciruzzi et $\mathrm{al}^{24}$.
Enstrom y Kabat ${ }^{25}$ no encontraron asociación del EHTA con muertes por cardiopatía coronaria, OR 0,94 (IC 0,85-1,05), pero consideraron sólo la exposición en el hogar y no la laboral, y tampoco evaluaron los cambios en la condición de fumadores-no fumadores de los cónyuges durante el seguimiento de casi 40 años (19601998).

Un trabajo reciente de Whincup et $\mathrm{al}^{26}$ demostró, en un seguimiento por 20 años de 2.105 varones no fumadores con medición de cotinina 
sanguínea, que a mayor concentración de ésta, mayor el riesgo de cardiopatía coronaria, con RR de 1,57 (IC 1,08-2,28), concluyendo que el exceso de riesgo puede llegar de 50 a $60 \%$.

Existen pocos trabajos que evalúan la asociación entre accidentes vasculares cerebrales (AVC) y la EHTA. En el estudio de Bonita et $\mathrm{al}^{27}, 521$ pacientes con AVC fueron comparados con 1.851 controles. Los hombres con EHTA tuvieron un riesgo 2,1 veces mayor (IC 95\% 1,33-3,32) de AVC que los no expuestos. Para las mujeres el OR fue 1,66 (IC 95\% 1,07-2,57).

En resumen, de acuerdo a los estudios experimentales y epidemiológicos, la EHTA produce marcados efectos dañinos en el sistema cardiovascular, aumentando el riesgo de enfermedad cardiovascular en un rango de 20 a $50 \%{ }^{9}$ y entre 25 a $35 \%$ el riesgo de eventos coronarios agudos $^{8}$.

\section{Efectos respiratorios}

Se ha demostrado que el humo de tabaco induce reacciones inflamatorias en la vía aérea y parénquima pulmonar de los fumadores activos y los efectos biológicos del HTA son similares a aquellos del humo de la corriente principal.

Se examinarán los efectos de la EHTA sobre síntomas respiratorios, función pulmonar, asma bronquial y EPOC.

\section{Sintomas Respiratorios}

Sintomas agudos:

Está bien establecido que la EHTA produce síntomas irritativos agudos de ojos, nariz, faringe y vía aérea inferior ${ }^{3,28}$. Los sujetos atópicos parecen ser más sensibles que los no atópicos ${ }^{9,29}$.

\section{Síntomas crónicos:}

Se ha demostrado aumento significativo en los síntomas respiratorios crónicos de adultos con EHTA, encontrándose también una relación dosis respuesta ${ }^{30,31}$.

Igual cosa ocurre en los niños cuando los padres fuman. En el meta- análisis de Strachan y Cook ${ }^{32}$ sobre enfermedades con sibilancias en niños pequeños, el fumar materno aumentó el riesgo de sibilancias, con OR de 1,98 (IC 95\% 1,71-2,3). En niños en edad escolar, los mismos autores ${ }^{33}$ resumieron los resultados de los estudios poblacionales caso-control: el riesgo de sibilancias se asoció con EHTA con OR de 1,24 (IC 95\% 1,10-1,34), para tos 1,40 (IC 95\% 1,27-1,53), para expectoración 1,35 (IC 95\% $1,13-1,62$ ) y para disnea 1,31 (IC 95\% 1,081,59). Los efectos fueron menores cuando la exposición se debió a uno de los padres, que cuando fue por ambos, indicando un efecto dosis-dependiente. El efecto del fumar materno fue mayor que el paterno, lo que puede explicarse por el mayor contacto del niño con la madre o por los efectos de fumar durante el embarazo. Resultados similares encontraron Peters et al en su meta-análisis ${ }^{34}$, donde el OR para cualquier síntoma respiratorio fue de 1,38 (IC 95\% 1,141,67) con dos o más fumadores en el hogar y de 1,15 (IC 95\% 1,01-1,31) para un sólo fumador.

\section{Función pulmonar}

Hay más de 20 estudios referidos al tema ${ }^{35}$, cuyos resultados han sido algo inconsistentes. Entre los estudios caso-control, 10 de ellos detectaron caída del $\mathrm{VEF}_{1}$, de índices de la pequeña vía aérea o de ambos parámetros, pero en 6 estudios no se encontraron asociaciones significativas. En los estudios longitudinales no se encontraron efectos de la EHTA sobre la función pulmonar en el tiempo ${ }^{36}$. Aparentemente, los efectos de la EHTA en la función pulmonar de adultos son dependientes de la dosis y pueden no observarse en bajas exposiciones.

En niños se ha demostrado que la EHTA altera la función pulmonar ${ }^{37}$, con directa relación con el número de cigarrillos fumados al día en el hogar. Los estudios de Mannino et $\mathrm{al}^{38}$ y Bono et al $^{39}$ encontraron relación entre niveles elevados de cotinina en niños y adolescentes, y disminución de la función pulmonar, aunque no está claro si ésta es permanente.

\section{Asma Bronquial}

Hay abundante evidencia del rol de la EHTA en el desarrollo y pronóstico del asma en niños, existiendo un número más limitado de estudios en adultos. Estos se pueden dividir en estudios etiológicos (inducción de asma) y estudios de la severidad y frecuencia de exacerbaciones en casos con la enfermedad establecida.

\section{Inducción de asma}

Se ha demostrado que la exposición tanto activa como pasiva al HTA se asocia con hiperreactividad bronquial, hecho característico del asma. La plausibilidad biológica ${ }^{35}$ de esta relación es fuerte, ya que: 1) La EHTA aumenta el riesgo de infecciones respiratorias repetidas en niños pequeños, lo que favorece el desarrollo de asma; 2) La EHTA causa hiperrespuesta de la vía aérea; 3) Puede aumentar el riesgo de atopia en los niños, con aumento de los niveles de IgE; y 4) El humo del tabaco causa inflamación de la vía aérea en fumadores activos y puede tener 
efecto similar, aunque de menor cuantía, en personas expuestas al HTA.

La EPA California $1997^{9}$ realizó un meta-análisis de 37 estudios, evaluando el efecto de la exposición post-natal al HTA en los hogares sobre el desarrollo de asma, bronquitis sibilante o sibilancias persistentes en niños y jóvenes menores de 18 años: el OR fue 1,44 (IC 95\% 1,27-1,64) para el diagnóstico clínico de asma y de 1,47 (IC 95\% 1,34-1,61) para bronquitis sibilante o sibilancias crónicas. Se concluye que es posible sostener una asociación causal entre EHTA y nuevos casos de asma en la infancia.

Desde 1996 a la fecha se han publicado aproximadamente otros 37 estudios que evalúan esta relación, de los cuales 31 sostienen la existencia de relación causal entre EHTA e inducción de asma durante la infancia. En la Tabla 5 se pue- den ver algunos de esos estudios. De ellos cabe destacar los estudios de cohorte (Tariq et $\mathrm{al}^{40}$, Oddy et $\mathrm{al}^{41}$, Ponsonby et $\mathrm{al}^{42}$, Jaakkola et $\mathrm{al}^{43}$ con seguimiento de miles de niños durante varios años, encontrándose en todos ellos una relación causal entre EHTA e inducción de asma en la infancia. De los 6 estudios en que no se encontró relación causal estadísticamente significativa, dos estudios (Cunningham et $\mathrm{al}^{44}$, Hu et $\mathrm{al}^{45}$ ) registraron, una relación causal importante entre exposición prenatal a HTA y aparición de nuevos casos de asma en la infancia.

En cuanto a la EHTA como factor de riesgo para asma en adultos, desde 1996 a la fecha se encontraron 9 estudios publicados: en 7 de ellos se concluyó que existe una relación causal entre EHTA e inducción de asma, destacando los estudios de cohorte prospectivos realizados por

Tabla 5. Algunos estudios de la Exposición al Humo de Tabaco Ambiental e Inducción de Asma Bronquial

\begin{tabular}{|c|c|c|}
\hline Estudio (referencia) & Tipo de estudio & OR o RR (IC 95\%) \\
\hline $\begin{array}{l}\text { Niños } \\
\text { Tariq et al (40) }\end{array}$ & Cohorte & $\begin{array}{l}\text { Prevalencia de asma: } \\
1 \text { año: } 2,5(1,7-3,7) \\
4 \text { años: } 1,2(0,3-2,7)\end{array}$ \\
\hline Oddy et al (41) & Cohorte & $1,27(1,04-1,55)$ \\
\hline Ponsonby et al (42) & Cohorte & Asma a los 7 años: $1,52(1,01-2,29)$ \\
\hline Jaakkola et al (43) & Cohorte & $\begin{array}{l}\text { Obstr. Bronquial 1,43 }(1,07-1,90) \\
\text { Asma Bronquial } 1,10(0,79-1,53)\end{array}$ \\
\hline Cunninghan et al (44) & Transversal & $\begin{array}{l}\text { Post natal 1,08 } \\
\text { Pre natal 2,7 (1,13-6,45) }\end{array}$ \\
\hline Hu et al (45) & Transversal & $\begin{array}{l}\text { Post natal } 0,8(0,5-1,5) \\
\text { Pre natal } 1,9(1,1-3,5)\end{array}$ \\
\hline Azizi et al (46) & Caso-control & $\begin{array}{l}\text { Primera hospitalización por crisis de } \\
\text { asma: } 1,91(1,13-3,21)\end{array}$ \\
\hline Agabiti et al (47) & Caso-control poblacional & $\begin{array}{l}\text { Asma a los 6-7 años } \\
\text { Madre fuma } 1,46(1,13-1,87) \\
\text { Padre fuma 1,26 }(1,01-1,58) \\
\text { Ambos fuman } 1,35(1,09-1,69)\end{array}$ \\
\hline $\begin{array}{l}\text { Adultos } \\
\text { Jaakkola et al (48) }\end{array}$ & Caso-control poblacional & $\begin{array}{l}\text { Casos nuevos de asma } \\
1,97(1,19-3,25)\end{array}$ \\
\hline Grear et al (49) & Cohorte & $\begin{array}{l}\text { Asma a los } 10 \text { años de seguimiento } \\
1,5(1,2-1,8)\end{array}$ \\
\hline Hu et al (50) & Cohorte & $\begin{array}{l}\text { Asma a los } 20-22 \text { años } \\
1,8(1,1-3,0)\end{array}$ \\
\hline Iribarren et al (51) & Transversal & $\begin{array}{l}\text { Asma diagnosticada por médicos } \\
1,22(1,11-1,34)\end{array}$ \\
\hline
\end{tabular}


Greer et $\mathrm{al}^{49}$ y Hu et $\mathrm{al}^{50}$ y el de Iribarren ${ }^{51}$, de tipo transversal, que estudió 47.721 adultos, encontrando OR de 1,22 para el riesgo de asma.

En conclusión, hay claras evidencias que apoyan la relación causal de la EHTA en el desarrollo de asma tanto en niños como en adultos.

\section{Gravedad y exacerbaciones de asma}

Las revisiones de EPA $1992^{6}$, y EPA California $1997^{9}$, concluyen que hay suficiente evidencia de la relación causal entre tabaquismo pasivo y nuevos episodios y aumento de la gravedad del asma en niños que ya presentan la enfermedad.

Catorce nuevos estudios desde $1997^{10}$ investigan la relación causal entre EHTA y exacerbación de asma en niños, de los cuales 9 son concluyentes en la existencia de esta relación, siendo los estudios longitudinales (Melen et $\mathrm{al}^{52}$, Schwartz et $\mathrm{al}^{53}$, Gilliland et $\mathrm{al}^{54}$ ) los más consistentes en demostrar efectos adversos.

El meta-análisis de Jaakkola et al ${ }^{55}$ (estudios entre 1966 y 2000), concluye que la EHTA aumenta la severidad del asma en niños, evaluada por la frecuencia e intensidad de las crisis de asma, el número de consultas a los servicios de urgencia, el uso de medicamentos de rescate y las hospitalizaciones por ataques de asma. Por otra parte, si la exposición postnatal al HTA se asocia a exposición prenatal, se encuentra aumento de la caída de la función pulmonar en niños asmáticos ( $\mathrm{Li}$ et $\left.\mathrm{al}^{56}\right)$.

En cuanto a la exacerbación de asma en adultos, desde 1997 a la fecha se han publicado al menos 7 estudios, encontrándose en todos ellos evidencia concluyente de que la EHTA causa exacerbación de asma en adultos, incluida la relación dosis-respuesta. Cabe destacar el estudio de Sippel et al ${ }^{57}$, (cohorte) en 619 personas entre 15 y 55 años, que encontró relación entre EHTA y peor evolución del asma y calidad de vida y aumento de hospitalizaciones (OR 2,34, IC 1,8-3,1) durante el seguimiento.

Por lo tanto, se concluye que las evidencias actuales apoyan fuertemente la relación causal entre EHTA y crisis de asma, tanto en niños como en adultos.

\section{Infecciones del tracto respiratorio inferior}

Existe convincente evidencia del tabaquismo pasivo y la susceptibilidad a infecciones respiratorias en niños. Koch et al ${ }^{58}$ demuestran riesgo atribuible de $47 \%$ para infecciones del tracto respiratorio inferior asociado al ser fumador pasivo en niños de 0-2 años. En el meta-análisis de Li et $\mathrm{al}^{59}$, la hospitalización por infecciones respiratorias bajas, se asoció a OR de 1,93 (IC
95\% 1,66-2,25), donde el riesgo era mayor para niños más pequeños con EHTA. En nuestro país, Oyarzún et $\mathrm{al}^{60}$ encontraron un OR 1,41 para la ocurrencia de infecciones pulmonares en lactantes de 4 a 12 meses con EHTA.

En adultos, la EHTA ha sido asociada con infecciones graves, como bacteriemia neumocócica, neumonía y meningitis, pero se requieren más estudios para tener conclusiones claras ${ }^{35}$.

\section{Otitis media}

La EHTA puede producir patología del oído medio por varios mecanismos biológicamente plausibles, en que la disfunción de la trompa de Eustaquio juega un rol central. Estos mecanismos son: disminución de la depuración ("clearance") muco-ciliar, disminución del lumen debido a hiperplasia adenoidal y/o edema de la mucosa, y aumento de las infecciones virales del tracto respiratorio superior ${ }^{10}$.

Por otra parte, los datos epidemiológicos sostienen una fuerte relación entre EHTA en el hogar y otitis media aguda, particularmente en niños menores de 2 años ${ }^{9,10}$.

Destacan los estudios de cohorte de Owen et $\mathrm{al}^{61}$ y Paradise et al ${ }^{62}$ que eliminaron el problema de los sesgos diagnósticos de otitis, utilizando otoscopía neumática y/o timpanometría. Los estudios de Etzel et al $^{63}$ y de Ilicali $^{64}$ utilizaron cotinina urinaria como marcador de exposición; éste último encontró un OR 2,29 (IC 95\% 1,084,85). En todos estos estudios se encontró una asociación significativa entre otitis media aguda $\mathrm{y}$ ser fumador pasivo.

\section{Enfermedad Pulmonar Obstructiva Crónica (EPOC)}

Existen pocos estudios que relacionan la EHTA y los estadios tardíos de la EPOC. La mayoría de ellos evalúan síntomas respiratorios crónicos, probablemente estadios tempranos de la enfermedad. Además, al existir diferentes definiciones epidemiológicas de EPOC se dificulta la evaluación de los estudios. Algunos de ellos han sugerido un efecto acumulativo con la EHTA desde la infancia a la adultez, en el hogar y los lugares de trabajo. El meta- análisis de Jaakkola ${ }^{35}$ demostró un riesgo aumentado de EPOC en los fumadores pasivos, que sería del 30 al 40\%, pero se requieren más estudios para llegar a afirmaciones más concluyentes.

\section{Cáncer y EHTA}

La IARC (2002) ha calificado al HTA como carcinógeno para las personas ${ }^{8}$. La plausibilidad biológica de ello deriva de la presencia de 
carcinógenos en el humo ambiental (Tabla 1) y a que no se ha determinado que exista en fumadores activos una dosis umbral carcinogénica. Además varios de los componentes del HTA tienen actividad genotóxica y capacidad de dañar el ADN.

Hay evidencias concluyentes sobre el rol patogénico de la EHTA en el desarrollo de cáncer pulmonar, mamario y de senos nasales, y sugerentes en cáncer cerebral y linfomas en niños, de acuerdo a la revisión de EPA California 2005. Se hará referencia a los tres primeros.

\section{Cáncer Pulmonar}

Desde el estudio de Hirayama et $\mathrm{al}^{65}$ en 1981 , se han publicado más de 50 estudios sobre fumar pasivo y cáncer pulmonar en no fumadores. La mayoría muestra riesgos aumentados, especialmente en personas con alta exposición. En la Tabla 6 se pueden ver algunos de ellos.

Hackshaw et al ${ }^{66}$ analizaron 37 estudios epidemiológicos sobre el riesgo de cáncer pulmonar en no fumadores: la revisión encontró que el exceso de riesgo es $24 \%$ (IC $95 \%$ de 13 a $36 \%$ ). Los ajustes por otros factores, tales como la dieta, no tuvieron importancia. Encontraron, además, carcinógenos específicos del tabaco en la sangre de los no fumadores, proporcionando claras evidencias del riesgo de fumar pasivamente. El estudio encontró, además, una relación dosis-respuesta entre el riesgo de cáncer pulmonar y el número de cigarrillos y años de EHTA. Johnson et $\mathrm{al}^{67}$ comunicaron aumento del riesgo de cáncer pulmonar después de ocho años de exposición laboral, OR 1,71 (IC 95\% 0,7-4,3). Por otra parte Wells ${ }^{69}$ analizó 14 estu- dios sobre el riesgo de EHTA en quienes nunca fueron fumadores y concluyó que en los fumadores pasivos en el lugar de trabajo aumenta el riesgo de cáncer pulmonar en un 39\%.

Boffetta et $\mathrm{al}^{70}$, en el mayor estudio europeo de EHTA (12 centros en 7 países), encontró un aumento pequeño del riesgo de cáncer pulmonar en no fumadores que trabajan en ambientes con humo o que viven con esposos que fuman. El mismo Boffetta realizó posteriormente un metaanálisis ${ }^{71}$, considerando 59 estudios, con 7.369 casos de cáncer pulmonar, encontrando un riesgo relativo de 1,25 (IC 95\% 1,15-1,37). Los resultados de acuerdo al tipo histológico, muestran un menor RR para adenocarcinoma que para carcinoma escamoso y de células pequeñas. En cuanto a la duración de la exposición, los resultados de dos grandes estudios multicéntricos (Boffetta et $\mathrm{al}^{70}$ y Fontham et $\mathrm{al}^{72} \mathrm{su}^{-}$ gieren una relación dosis-respuesta, de acuerdo a la dosis acumulativa. Se ha demostrado además que los ex-fumadores son más susceptibles que quienes nunca han sido fumadores, para desarrollar cáncer pulmonar con la EHTA ${ }^{73}$.

El Comité Científico del Reino Unido de Tabaco o Salud ${ }^{7}$ después de revisar las evidencias concluyó: "los no fumadores con EHTA en el largo plazo tienen un aumento del riesgo de cáncer pulmonar entre 20 a 30\%." El informe de la EPA California $1997^{9}$ llegó a similares conclusiones: "tomados en su conjunto los recientes estudios proporcionan evidencia adicional de que la EHTA está causalmente asociada con cáncer pulmonar, con $20 \%$ de exceso de riesgo en no fumadores", lo cual fue reconfirmado en el informe de la misma agencia de 2005.

Tabla 6. Algunos estudios de la Exposición al Humo de Tabaco Ambiental y Cáncer Pulmonar

\begin{tabular}{lll}
\hline Estudio (referencia) & Tipo de estudio & OR o RR (IC 95\%) \\
\hline Hackshaw et al (66) & Meta análisis & $1,24(1,13-1,36)$ \\
Johnson et al (67) & Caso control poblacional & Exposición laboral: $1,71(0,7-4,3)$ \\
Wang et al (68) & Caso control poblacional & Exposición laboral: $1,51(0,9-2,7)$ \\
Boffetta et al (70) & Caso control multicéntrico & Exposición laboral y en hogar: \\
Boffetta et al (71) & Meta análisis & $1,54(0,97-2,44)$ \\
Fontham et al (72) & Caso control multicéntrico & $1,25(1,15-1,37)$ \\
Vineis et al (73) & Caso control poblacional & $1,74(1,14-2,65)$ \\
\hline
\end{tabular}




\section{Cáncer de senos nasales}

Ya en 1997 el informe de la EPA-California concluyó que existe una asociación positiva entre la EHTA y cáncer de senos nasales (CSN) en no fumadores. Esto ha sido corroborado en estudios posteriores, siendo el más importante el de Yuan et al ${ }^{74}$ realizado en Shangai, que mostró elevado riesgo de desarrollo de CSN con OR 1,95 (IC 95\% 1,18-3,21) en mujeres, pero no en hombres, OR 1,21 (IC 0,62-2,68). Este mismo estudio demostró, además, evidencia de relación dosis-respuesta entre la EHTA y riesgo de CSN.

\section{Cáncer de mama}

Diversos trabajos de década del '80 y comienzos de los 90 (Hirayama ${ }^{75}$, Sandler ${ }^{76}$ y Smith $^{77}$ ) habían mostrado una tendencia al aumento del riesgo de cáncer de mama en mujeres con EHTA, tendencia no concluyente. Publicaciones posteriores (Johnson ${ }^{78}$, Kropp ${ }^{79}$ ) han demostrado riesgos estimados promedios de 1,40 (IC 95\% 1,17-1,68) confirmando una relación positiva entre fumar pasivo y cáncer de mama, particularmente en mujeres premenopáusicas y expuestas precozmente en la vida al HTA.

\section{Efectos de la EHTA en la salud reproductiva}

Los componentes del HTA pueden difundir a la placenta de las madres no fumadoras expuestas. Se han encontrado compuestos carcinogénicos, mutagénicos y teratogénicos en placenta, líquido amniótico y sangre del cordón umbilical de fetos humanos y animales de madres fumadoras pasivas. Además se ha observado un aumento de los glóbulos rojos circulantes en los fetos de madres expuestas al humo de tabaco, lo que sería una señal de hipoxia, producida probablemente por vasoconstricción placentaria inducida por la nicotina ${ }^{80}$.

Existe evidencia que sugiere que la EHTA afecta la fertilidad y fecundidad en la mujer y produce desórdenes en los ciclos menstruales, así como aumento de dismenorrea. En mujeres no fumadoras cuyos cónyuges fuman, la edad de la menopausia disminuye en promedio 2 años (OR 2,1 (IC 95\% 1,04-4,5)) según Everson et $\mathrm{al}^{81}$. En hombres no existe aún evidencia concluyente sobre alteración del líquido seminal en fumadores pasivos, que se sabe que se produce en el fumar activo. Sin embargo, existen hallazgos de cotinina en el semen de no fumadores con EHTA $^{80}$.

El feto está también expuesto a los productos del humo del tabaco cuando la mujer embaraza- da se expone. La mayor evidencia de los efectos en la salud reproductiva de la EHTA está en el efecto sobre el peso de nacimiento, que decrece entre 20 a $100 \mathrm{~g}^{10}$. El meta-análisis de Windham et $\mathrm{al}^{82}$, (25 estudios evaluados) encontró disminución del peso de nacimiento en el rango de 15 a $60 \mathrm{~g}$, con aumento de los recién nacidos de bajo peso de nacimiento $(<2.500 \mathrm{~g}$ o pequeños para la edad gestacional) entre 10 a $30 \%$.

Existe evidencia sugerente que también se incrementa el riesgo de partos de pretérmino, con OR entre 1,29 y $3,8^{10}$. En abortos espontáneos, fecundidad y defectos de nacimiento la evidencia es débil y no concluyente ${ }^{80}$.

Ha sido difícil diferenciar si los efectos dañinos se deben al efecto directo del fumar activo en el hombre y su alteración en la fertilidad, o a la exposición pasiva de la mujer. Se necesitan más estudios en mujeres embarazadas con EHTA en el trabajo, pero que vivan con no fumado$\mathrm{res}^{80}$, para eliminar este factor confundente.

\section{Síndrome de Muerte Súbita Infantil (SMSI)}

Ha existido confusión entre los efectos de la exposición pre y postnatal al HTA en la incidencia del SMSI. Esta ha sido aclarada en el metaanálisis de Anderson y $\operatorname{Cook}^{83}$, que incluyó 4 estudios que comunican los efectos de la EHTA postnatal, controlando la exposición materna prenatal, con OR de 1,94 (IC 1,55-2,43). Además, los hallazgos de elevados niveles de cotinina y/o nicotina $^{84,85}$ en tejidos de niños fallecidos por SMSI comparados con otras causas de muerte, apoya el efecto postnatal de la EHTA. Esto se infiere también de los hallazgos del aumento del riesgo para SMSI asociado al padre fumador, en los estudios de Brooke et al ${ }^{86} \mathrm{y}$ Mitchell et $\mathrm{al}^{87}$.

Brooke et al ${ }^{86}$ encontró que el riesgo asociado al fumar de ambos padres es 5,19 (IC 95\% 2,26-11,91). Si sólo la madre fuma el riesgo es $5,05(1,85-13,77)$ y si sólo el padre es fumador, es 2,12 (0,99-4,56). Este estudio, más los de Klonoff-Cohen ${ }^{88}$ y Mitchell et $\mathrm{al}^{87}$, apoyan una relación dosis respuesta entre el número de cigarrillos fumados en el hogar y el riesgo de SMSI.

Esta asociación ha sido fortalecida por la evidencia de varios mecanismos por los cuales el tabaco altera las regiones cerebrales responsables de controlar la respuesta cardiorrespiratoria, los receptores muscarínicos y adrenérgicos en el corazón, el tamaño de la vía aérea (más su inflamación, alergia y enfermedades infecciosas) y la capacidad de respuesta frente a la hipoxia inducida por apnea o humo de tabaco ${ }^{10}$. 
En conclusión, los datos existentes indican una relación causal entre la exposición postnatal y el riesgo de SMSI. Resumiendo los diferentes trabajos, se estima aumento del riesgo con OR $2,5^{9,10}$.

\section{CONCLUSIONES Y COMENTARIOS}

De acuerdo a la información científica disponible, se concluye que la EHTA daña la salud de los no fumadores, aumentando el riesgo de contraer enfermedades como: cáncer pulmonar, mamario y de senos nasales; cardiopatía coronaria; inducción y exacerbación de asma en niños y adultos; otitis media en niños; síntomas y enfermedades respiratorias infecciosas en niños; disminución del peso de nacimiento en recién nacidos con EHTA durante el embarazo, aumento de los recién nacidos de bajo peso y aumento del riesgo de SMSI en niños.

Por lo tanto, el HTA es uno de los contaminantes de los ambientes interiores más extendido y dañino para la salud de los no fumadores, tanto en los hogares como en los lugares de trabajo. Ésta es un área en que las compañías de tabaco han intentado desvirtuar la información científica, poniendo un manto de dudas sobre ella ${ }^{89-90}$. Como los datos disponibles son categóricos, el corolario evidente y obvio es la necesidad de instaurar medidas de política pública para proteger la salud de los no fumadores, restringiendo fumar en los lugares de trabajo y en los espacios públicos.

La restricción de fumar en el lugar de trabajo es una forma eficaz de reducir la EHTA, ya que la separación de espacios no aislados ambientalmente, por ejemplo en restaurantes, no protege realmente ${ }^{91}$. En nuestro país se está desarrollando el Programa Ambientes Libres del Humo de Tabaco, desde hace cuatro años ${ }^{92}$, a través del cual más de 2.000 instituciones se han acreditado como ambientes libres del humo de tabaco. De acuerdo a un estudio reciente ${ }^{93}$, el $94,7 \%$ de los no fumadores y el $82 \%$ de los fumadores aprueban las restricciones para fumar en el sector salud, y el $95,7 \%$ de los no fumadores y el $86,6 \%$ de los fumadores las aprueban en el sector educación ${ }^{94}$, lo que hace suponer que las medidas restrictivas serían aceptadas por la población, especialmente en el ámbito laboral.

En un momento en que en el país se está discutiendo una nueva ley para el control del consumo de tabaco, disponer de adecuada información científica es fundamental para una buena toma de decisiones.

\section{BIBLIOGRAFÍA}

1.- www.who.int/tobacco/en/

2.- Ministerio de Salud. Departamento de Estadísticas e Información en Salud.

3.- US Department of Health and Human Services. The health consequences of involuntary smoking. A Report of the Surgeon General. Washington D.C. US Government Printing Office 1986.

4.- US National Research Council, Committee on Passive Smoking. Environmental tobacco smoke: Measuring exposures and assessing health effects. Washington D.C. National Academy Press. 1986.

5.- Effects of passive smoking on health National Health and Medical Research Council. Australian Government Publishing Service, 1987.

6.- US Environmental Protection Agency. Respiratory health effects of passive smoking: lung cancer and other disorders. Washington, DC: US Government Printing Office; 1992; EPA/600/006F.

7.- UK Scientific Committee on Tobacco and Health. Report of the Scientific Committee on Tobacco and Health. The Stationery Office. March 1998.

8.- International Agency for Research on Cancer: Tobacco smoke and involuntary smoking: IARC Monographs on the Evaluation of the Carcinogenic Risk of Chemicals to Humans, vol. 83. Lyon, International Agency for Research on Cancer, 2002.

9.- US Department of Health and Human Services: Smoking and tobacco control. National Cancer Institute (NCI): Health effects of exposure to environmental tobacco smoke: The Report of the California Environmental Protection Agency. Monograph No10 (Bethesda, MD): National Institutes of Health, 1999.

10.- Report of the California Environmental Protection Agency. 2005. www.arb.ca.gov/toxics/ets/dreport/ dreport.htm

11.- www.int/tobacco/framework/final_text/en/

12.- SAMET J M, WANG S S. Environmental Tobacco Smoke. En Environmental Toxicants: Human Exposures and their Health Effects. M. Lippman Editor. Nueva York. Wiley and Sons 1999.

13.- ROSENBERG J, BENOWITZ N L, JACOB P, WILSON K M. Disposition kinetics and effects of intravenous nicotine. Clin Pharmacol Ther 1980; 28: 517-22.

14.- BENOWITZ N L, KUYT F, JACOB P, JONES R T, OSMAN A L. Cotinine disposition and effects. Clin Pharmacol Ther 1983; 34: 604-11.

15.- JARVIS M J, RUSSEL M A. Measurement and estimation of smoke dosage to non-smokers from environmental tobacco smoke. Eur J Respir Dis 1984; 133: 68-75.

16.- Action on Smoking and Heath (ASH) briefing on passive smoking. ASH-UK, 2000.

17.- GLANTZ S A, PARMLEY W W. Passive smoking and heart disease epidemiology, physiology and biochemistry. Circulation 1991; 83: 1-12.

18.- GLANTZ S A, PARMLEY W W. Passive smoking and heart disease. JAMA 1995; 273: 1047-53.

19.- JOUSILAHTI P, PATJA K, SALOMAA V. Environmental tobacco smoke and the risk of cardiovascular disease. Scand J Work Environ Health 2002; 28 suppl 2: 41-51.

20.- WELLS A J. Heart disease from passive smoking in the work place. J Am Coll Cardiol 1998; 31: 1-9.

21.- LAW M R, MORRIS J K, WALD N J. Environmental 
tobacco smoke exposure and ischemic heart disease: an evaluation of the evidence. Brit Med J 1997; 315: 973-80.

22.- HE J, VUPPUTURI S, ALLEN K, PREROST M, HUGHES J, WHELTON P. Passive Smoking and the Risk of Coronary Heart Disease - A Meta-Analysis of Epidemiologic Studies. New Engl J Med 1999; 340: 920-6.

23.- THUN M, HENLEY J, APICELLA L. Epidemiologic studies of fatal and non fatal cardiovascular diseases and ETS exposure from spousal smoking. Environ Health Perspect 1999; 107 (suppl 6): 920-6.

24.- CIRUZZI M, PRAMPARO P, ESTEBAN O, ROZLOSNIK J, TARTAGLIONE J, ABECASIS B, et al. Case-control study of passive smoking at home and risk of acute myocardial infarction. Argentine FRICAS Investigators. Factores de Riesgo Coronario en América del Sur. J Am Coll Cardiol 1998; 31: $797-$ 803.

25.- ENSTROM J, KABAT G. Environmental tobacco smoke and tobacco related mortality in a prospective study of Californians, 1960-1998. Brit Med J 2003; 326: 1057-66.

26.- WHINCUP P, GILG J, EMBERSON J, JARVIS M, FEYERABEND C, BRYANT A, et al. Passive smoking and risk of coronary heart disease and stoke: prospective study with cotinine measurement. Brit Med J 2004; 329: 200-5.

27.- BONITA R, DUNCAN J, TRUELSEN T, JACKSON R T, BEAGLEHOLE R. Passive smoking as well as active smoking increases the risk of acute stroke. Tob Control 1999; 8: 156-60.

28.- TREDANIEL J, BOFFETTA P, SARACCI R, HIRSCH A. Exposure to environmental tobacco smoke and adult non neoplastic respiratory diseases. Eur Respir J 1994; 7: 173-85.

29.- BASCOM R, KULLE T, KAGEY-SOBOTKA A, PROUD D. Upper respiratory tract environmental tobacco smoke sensitivity. Am Rev Respir Dis 1991; 143: $1304-11$.

30.- LEUENBERGER P, SCHWARTZ J, ACKERMANNLIEBRICH U, BLASER K, BOLOGNINI G, BONGARD J P, et al. Passive smoking exposure in adults and chronic respiratory symptoms. Am J Respir Crit Care Med 1994; 150: 1222-8.

31.- JAAKKOLA M S, JAAKKOLA J J K, BECKLAKE M R, ERNST P. Effect of passive smoking on the development on respiratory symptoms in young adults: an 8-year longitudinal study. J Clin Epidemiol 1996; 49: 581-6.

32.- STRACHAN D P, COOK D G. Parental smoking and lower respiratory illness in infancy and early childhood. Thorax 1997; 52: 905-14.

33.- COOK D G, STRACHAN D P. Summary of effects of parental smoking on the respiratory health of children and implications for research. Thorax 1999; 54: 357 66.

34.- PETERS J, MCCABE C J, HEDLEY A J, LAM T H, WONG C M. Economic burden of environmental tobacco smoke on Hong Kong families: scale and impact. J Epidemiol Community Health 1998; 52: 53-8.

35.- JAAKKOLA M, JAAKKOLA J. Effects of environmental tobacco smoke on the respiratory health of adults. Scand J Work Environ Health 2002; 28: 52 70

36.- CAREY I M, COOK D G, STRACHAN D P. The effects of environmental tobacco smoke exposure on lung function in a longitudinal study of British adults. Epidemiology 1999; 10: 319-26.

37.- NUHOGLU C, GURUL M, NUHOGLU Y, KARATOPRAK N, SONMEZ EO, YAVRUCU S, OZGUNER A. Effects of passive smoking on lung function in children. Pediatr Int 2003; 45: 426-8.

38.- MANNINO D M, MOORMAN J E, KINGSLEY B, ROSE D, REPACE J. Health effects related to environmental tobacco smoke exposure in children in the United States: data from the Third National Health and Nutrition Examination Survey. Arch Pediatr Adolesc Med 2001; 155: 36-41.

39.- BONO R, NEBIOLO F, BUGIANI M, MEINERI V, SCURSATONE E, PICCIONI P, et al. Effects of tobacco smoke exposure on lung growth in adolescents. J Expo Anal Environ Epidemiol 1998; 8: 335-45.

40.- TARIQ S, MATTEWS S, HAKIM E, STEVENS M, ARSHAD S, HIDE D. The prevalence of and risk factors for atopy in early childhood: a whole population birth cohort study. J Allergy Clin Immunol 1998; 101: 587-93.

41.- ODDY W, HOLT P, SLY P, READ A, LANDAU L, STANLEY F et al. Association between breast feeding and asthma in 6 year old children: findings of a prospective birth cohort study. Brit Med J 1999; 319 (7213): 815-9.

42.- PONSONBY A, COUPER D, DWYER T, CARMICHAEL A, KEMP A, COCHRANE J. The relation between infant indoor environment and subsequent asthma. Epidemiology 2000; 11: 128-35.

43. JAAKKOLA J, NAFSTAD P, MAGNUS P. Environmental tobacco smoke, parental atopy and childhood asthma. Environ Heath Perspect 2001; 109: 579-82.

44.- CUNNINGHAM J, O CONNOR G, DOCKERY D, SPEIZER F. Environmental tobacco smoke, wheezing and asthma in children in 24 communities. Am J Respir Crit Care Med 1996; 153: 218-24.

45.- HU F, PERSKY V, FLAY B, ZELLI A, COOKSEY J, RICHARDSON J. Prevalence of asthma and wheezing in public schoolchildren: association with maternal smoking during pregnancy. Ann Allergy Asthma Immunol 1997; 79: 80-4.

46.- AZIZI B, ZULKIFLI H, KASIM S. Indoor air pollution and asthma in hospitalized children in a tropical environment. J Asthma 1995; 32 : 413-8.

47.- AGABITI N, MALLONE S, FORASTIERE F, CORBO G, FERRO S, RENZONI E, et al. The impact of parental smoking on asthma and wheezing. SIDRIA Collaborative Group. Studi Italiani sui Disturbi Respiratori nell'Infanzia e l'Ambiente. Epidemiology 1999; 10: 692-8.

48.- JAAKKOLA M, PIIPARI R, JAAKKOLA N, JAAKKOLA J. Environmental tobacco smoke and adult-onset asthma, a population-based case-control study. Am J Public Health 2003; 93: 2055-60.

49.- GREER J, ABBEY D, BURCHETTE R. Asthma related to occupational and ambient air pollutants in non smokers. J Occup Med 1993; 35: 909-15.

50.- HU F, PERSKY V, FLAY B, RICHARDSON J. An epidemiological study of asthma prevalence and related factors among young adults. J Asthma 1997; 34: 67 76.

51.- IRIBARREN C, FRIEDMAN G D, KLATSKY A L, EISNER M D. Exposure to environmental tobacco smoke: association with personal characteristics and self reported health conditions. J Epidemiol Community Health 2001; 55: 721-8. 
52.- MELEN E, WICKMAN M, NORDVALL S, VAN HAGE-HAMSTEN M, LINDFORS A. Influence of early and current environmental exposure factors on sensitization and outcome of asthma in pre-school children. Allergy 2001; 56: 646-52.

53.- SCHWARTZ J, TIMONEN K, PEKKANEN J. Respiratory effects environmental tobacco smoke in a panel study of asthmatic and symptomatic children. Am J Respir Crit Care Med 2000; 161: 802-6.

54.- GILLILAND F, LI Y, PETERS J. Effects of maternal smoking during pregnancy and environmental tobacco smoke on asthma and wheezing in children. Am J Respir Crit Care Med 2001; 163: 429-36.

55.- JAAKKOLA J J, JAAKKOLA M S. Effects of environmental tobacco smoke on the respiratory health of children. Scand J Work Environ Health 2002; 28: 71-83.

56.- LI Y F, GILLILAND F D, BERHANE K, MCCONNELL R, GAUDERMAN W J, RAPPAPORT $\mathrm{E} B$, et al. Effects of in utero and environmental tobacco smoke exposure on lung function in boys and girls with and without asthma. Am J Respir Crit Care Med 2000; 162: 2097-104.

57.- SIPPEL J M, PEDULA K L, VOLLMER W M, BUIST A S, OSBORNE M L. Associations of smoking with hospital-based care and quality of life in patients with obstructive airway disease. Chest 1999; 115: 691-6.

58.- KOCH A, MOLBAK K, HOMOE P, SORENSEN P, HJULER T, OLESEN M E, PEJL J, PEDERSEN F K, OLSEN O R, MELBYE M. Risk factors of acute respiratory tract infections in young Greenlandic children. Am J Epidemiol 2003; 158: 374-84.

59.- LI J S, PEAT J K, XUAN W, BERRY G. Meta-analysis on the association between environmental tobacco smoke (ETS) exposure and the prevalence of lower respiratory tract infection in early childhood. Pediatr Pulmonol 1999; 27: 5-13.

60.- OYARZÚN M, PINO P, ORTIZ J, OLAETA I. Effect of atmospheric pollution on the respiratory system. Biol Res 1998; 31: 361-6.

61.- OWEN M J, BALDWIN C D, SWANK P R, PANNU A R, JOHNSON D L, HOWIE V M. Relation of infant feeding practices, cigarette smoke exposure, and group child care to the onset and duration of otitis media with effusion in the first two years of life. J Pediatr 1993; 123: 702-11.

62.- PARADISE J L, ROCKETTE H E, COLBORN D K, BERNARD B S, SMITH C G, KURS-LASKY M, et al. Otitis media in 2253 Pittsburgh-area infants: prevalence and risk factors during the first two years of life. Pediatrics 1997; 99: 318-33.

63.- ETZEL R A, PATTISHALL E N, HALEY N J, FLETCHER R H, HENDERSON F W. Passive smoking and middle ear effusion among children in day care. Pediatrics 1992; 20 (Pt 1): 228-32.

64.- ILICALI O C, KELES N, DE ER K, SA UN O F, GULDIKEN Y. Evaluation of the effect of passive smoking on otitis media in children by an objective method: urinary cotinine analysis. Laryngoscope 2001; 111: 163-7.

65.- HIRAYAMA T. Non Smoking wives of heavy smokers have a higher risk of lung cancer: a study from Japan. Brit Med J 1981; 282 (6259): 183-5.

66.- HACKSHAW A K, LAW M R, WALD N J. The accumulated evidence on lung cancer and environmental tobacco smoke. Brit Med J 1997; 315: 9808.

67.- JOHNSON K C, HU J, MAO Y. Lifetime residential and workplace exposure to environmental tobacco smoke and lung cancer in never-smoking women, Canada 1994-97. Intl J Cancer 2001; 93: 902-6.

68.- WANG S, HU Y, WU Y, LI X, CHI G, CHEN Y, et al. A comparative study of the risk factors for lung cancer in Guaandong, China. Lung Cancer 1996; 14 Suppl 1: 99-105.

69.- WELLS A J. Lung cancer from passive smoking at work. Am J Pub Health 1998; 88: 1025-9.

70.- BOFFETTA P, AGUADO A, AHRENS W, BENHAMOU E, BENHAMOU S, DARBY S C, et al. Multicenter case-control study of exposure to environmental tobacco smoke and lung cancer in Europe. J Natl Cancer Inst 1998; 90: 1440-50.

71.- BOFFETTA P. Involuntary smoking and lung cancer. Scand J Work Environ Health 2002; 28 suppl 2: 3040.

72.- FONTHAM E T, CORREA P, REYNOLDS P, WUWILLIAMS A, BUFFLER P A, GREENBERG R S, et al. Environmental tobacco smoke and lung cancer in nonsmoking women: a multicenter study. JAMA 1994; 271: 1752-9.

73.- VINEIS P, AIROLDI L, VEGLIA F, OLGIATI L, PASTORELLI R, AUTRUP H, et al. Environmental tobacco smoke and risk of respiratory cancer and chronic obstructive pulmonary disease in former smokers and never smokers in the EPIC prospective study. Brit Med J,_doi:10.1136/bmj.38327.648472.82 (published 1 February 2005).

74.- YUAN J-M, WANG W-L, XIANG Y-B, GAO Y-T, ROSS R, YU M. Non dietary risk factors for nasopharyngeal carcinoma in Shanghai, China. Intl J Cancer 2000; 85: 364-9.

75.- HIRAYAMA T. Cancer mortality in non smoking women with smoking husbands based on a large scale cohort study in Japan. Prev Med 1984; 13: 680-90.

76.- SANDLER D P, EVERSON R B, WILCOX A J. Passive smoking in adulthood and cancer risk. Am J Epidemiol 1985; 121: 37-48.

77.- SMITH S J, DEACON J M, CHILVERS C E. Alcohol, smoking, passive smoking and caffeine in relation to breast cancer risk in young women. UK National CaseControl Study Group. Br J Cancer 1994; 70: 112-9.

78.- JOHNSON K C, HU J, MAO Y. Passive and active smoking and breast cancer risk in Canada, 1994-97. The Canadian Cancer Registries Epidemiology Research Group. Cancer Causes Cont 2000; 11: 21121.

79.- KROPP S, CHANG-CLAUDE J. Active and passive smoking and risk of breast cancer by age 50 years among German women. Am J Epidemiol 2002; 156: 616-26.

80.- LINDBOHM M-L, SALLMÉN M, TASKINEN H. Effects of exposure to environmental tobacco smoke on reproductive health. Scand J Work Environ Health 2002; 28 suppl 2: 84-96.

81.- EVERSON R B, SANDLER D P, WILCOX A J, SCHREINEMACHERS D, SHORE D L, WEINBERG C. Effect of passive exposure to smoking on age at natural menopause. Brit Med J 1986; 293 (6550): 792.

82.- WINHDAM G C, EATON A, HOPKINS B. Evidence for an association between environmental tobacco smoke exposure and birth weight: a meta-analysis and new data. Pediatr Perinat Epidemiol 1999; 13: 35-57.

83.- ANDERSON H R, COOK D G. Passive smoking and sudden infant death syndrome: review of the epidemiological evidence. Thorax 1997; 52: 1003-9.

84.- MILERAD J, VEGE A, OPDAL S H, ROGNUM T O. 
Objective measurements of nicotine exposure in victims of sudden infant death syndrome and in other unexpected child deaths. J Pediatr 1998; 133: 232-6.

85.- MC MARTIN K I, PLATT M S, HACKMAN R, KLEIN J, SMIALEK J E, VIGORITO R et al. Lung tissue concentrations of nicotine in sudden infant death syndrome (SIDS). J Pediatr 2002; 140: 205-9.

86.- BROOKE H, GIBSON A, TAPPIN D, BROWN H. Case-control study of sudden infant death syndrome in Scotland, 1992-5. Brit Med J 1997; 314 (7093): 1516-20.

87.- MITCHELL E A, TUOHY P G, BRUNT J M, THOMPSON J M, CLEMENTS M S, STEWART A $\mathrm{W}$, et al. Risk factors for sudden infant death syndrome following the prevention campaign in New Zealand: a prospective study. Pediatrics 1997; 100: 835-40.

88.- KLONOFF-COHEN H S, EDELSTEIN S L, LEFKOWITZ E S, SRINIVASAN I P, KAEGI D, CHANG $\mathrm{J} \mathrm{C}$, et al. The effects of passive smoking and tobacco exposure through breast milk on sudden infant death syndrome. JAMA 1995; 173: 795-8.
89.- BARNOYA J, GLANTZ S. Tobacco industry success in preventing regulation of secondhand smoke in Latin America: the "Latin Project". Tobacco Control 2002; 11: 305-14.

90.- BARNES D E, BERO L A. Why review articles on the health effects of passive smoking reach different conclusions. JAMA 1998; 279: 1566-70.

91.- NAVAS-ACIEN A, PERUGA A, BREYSSE P, ZAVALETA A, BLANCO-MARQUIZO A, PITARQUE R, et al. Secondhand Tobacco Smoke in Public Places in Latin America, 2002-2003. JAMA 2004; 291: 2741-5.

92.- Ministerio de Salud. División de Atención Primaria. Departamento de Promoción de la Salud. Salud Sin Tabaco. Guía Técnica - Metodológica. Programa Ambientes Libres del Humo de Tabaco. 2001.

93.- BELLO S, SOTO M, MICHALLAND S, SALINAS J. Encuesta nacional de tabaquismo en funcionarios de salud. Rev Méd Chile 2004; 132: 223-32.

94.- BELLO S, MICHALLAND M, SOTO M, SALINAS J. Tabaquismo en funcionarios de educación. Rev Chil Enf Respir 2004; 20: 131-8. 